\title{
Convergent expansions of the Bessel functions in terms of elementary functions
}

\author{
José L. López ${ }^{1}$ \\ ${ }^{1}$ Dpto. de Ingeniería Matemática e Informática and INAMAT, Universidad Pública de Navarra \\ e-mail: jl.lopez@unavarra.es
}

\begin{abstract}
We consider the Bessel functions $J_{\nu}(z)$ and $Y_{\nu}(z)$ for $\Re \nu>-1 / 2$ and $\Re z \geq 0$. We derive a convergent expansion of $J_{\nu}(z)$ in terms of the derivatives of $(\sin z) / z$, and a convergent expansion of $Y_{\nu}(z)$ in terms of derivatives of $(1-\cos z) / z$, derivatives of $\left(1-e^{-z}\right) / z$ and $\Gamma(2 \nu, z)$. Both expansions hold uniformly in $z$ in any fixed horizontal strip and are accompanied by error bounds. The accuracy of the approximations is illustrated with some numerical experiments.
\end{abstract}

2010 AMS Mathematics Subject Classification: 33C10; 41A58.

Keywords \& Phrases: Bessel functions; convergent expansions; error bounds; uniform expansions.

\section{Introduction}

The power series expansions of the Bessel function $J_{\nu}(z)$ is well known [4, Sec. 2, eq. $(10.2 .2)]$,

$$
\left(\frac{2}{z}\right)^{\nu} J_{\nu}(z)=\sum_{k=0}^{n-1} \frac{\left(-z^{2} / 4\right)^{k}}{k ! \Gamma(\nu+k+1)}+R_{n}^{(J, 0)}(\nu, z)
$$

For non integer $\nu$, the power series expansions of the Bessel function $Y_{\nu}(z)$ follows straightforwardly from this formula and the connection formula $Y_{\nu}(z)=\left[J_{\nu}(z) \cos (\pi \nu)-J_{-\nu}(z)\right] / \sin (\pi \nu)$ [4, Sec. 2, eq. (10.2.3)]:

$$
\left(\frac{2}{z}\right)^{\nu} Y_{\nu}(z)=\frac{1}{\sin (\pi \nu)} \sum_{k=0}^{n-1} \frac{\left(-z^{2} / 4\right)^{k}}{k !}\left[\frac{\cos (\pi \nu)}{\Gamma(\nu+k+1)}-\frac{(2 / z)^{2 \nu}}{\Gamma(k+1-\nu)}\right]+R_{n}^{(Y, 0)}(\nu, z) .
$$


For integer $\nu$, the power series expansions of $Y_{\nu}(z)$ follows from the limiting value of this formula [4, Sec. 2, eq. (10.2.4)]. The asymptotic expansions of the Bessel function $J_{\nu}(z)$ in terms of inverse powers of $z$ is also well known [4, Sec. 17, eq. (10.17.3)],

$$
\sqrt{\frac{\pi z}{2}} J_{\nu}(z)=\cos \left(z-\frac{\nu \pi}{2}-\frac{\pi}{4}\right) \sum_{k=0}^{n-1} \frac{a_{2 k}(\nu)}{\left(-z^{2}\right)^{k}}-\sin \left(z-\frac{\nu \pi}{2}-\frac{\pi}{4}\right) \sum_{k=0}^{n-1} \frac{a_{2 k+1}(\nu)}{z\left(-z^{2}\right)^{k}}+R_{n}^{(J, \infty)}(\nu, z),
$$

with $a_{0}(\nu)=1$ and, for $k=1,2,3, \ldots$,

$$
a_{k}(\nu)=\frac{\left(4 \nu^{2}-1^{2}\right)\left(4 \nu^{2}-3^{2}\right) \cdots\left(4 \nu^{2}-(2 k-1)^{2}\right)}{k ! 8^{k}} .
$$

The asymptotic expansions of $Y_{\nu}(z)$ is similar [4, Sec. 17, eq. (10.17.4)]:

$$
\sqrt{\frac{\pi z}{2}} Y_{\nu}(z)=\sin \left(z-\frac{\nu \pi}{2}-\frac{\pi}{4}\right) \sum_{k=0}^{n-1} \frac{a_{2 k}(\nu)}{\left(-z^{2}\right)^{k}}+\cos \left(z-\frac{\nu \pi}{2}-\frac{\pi}{4}\right) \sum_{k=0}^{n-1} \frac{a_{2 k+1}(\nu)}{z\left(-z^{2}\right)^{k}}+R_{n}^{(Y, \infty)}(\nu, z),
$$

A good property of these expansions is that they are given in terms of elementary functions. On the other hand, they have an inconvenience: they are not uniformly valid for all values of $z$; the remainders $R_{n}^{(J, 0)}(\nu, z)$ and $R_{n}^{(Y, 0)}(\nu, z)$ are unbounded for large values of $|z|$, whereas the remainders $R_{n}^{(J, \infty)}(\nu, z)$ and $R_{n}^{(Y, \infty)}(\nu, z)$ are unbounded for small values of $|z|$.

In this paper we derive convergent expansions of $J_{\nu}(z)$ and $Y_{\nu}(z)$ in terms of elementary functions that hold uniformly in $z$ in any fixed horizontal strip. The starting point is the following Poisson's integral representation of the Bessel functions [4, Sec. 9, eqs. (10.9.4) and (10.9.5)], valid for $\Re \nu>-1 / 2$ :

$$
\begin{array}{rlrl}
J_{\nu}(z) & =\frac{2(z / 2)^{\nu}}{\sqrt{\pi} \Gamma(\nu+1 / 2)} \bar{J}_{\nu}(z) ; & \bar{J}_{\nu}(z):=\int_{0}^{1}\left(1-t^{2}\right)^{\nu-1 / 2} \cos (z t) d t \\
Y_{\nu}(z) & =\frac{2(z / 2)^{\nu}}{\sqrt{\pi} \Gamma(\nu+1 / 2)}\left(Y_{\nu}^{1}(z)-Y_{\nu}^{2}(z)\right) ; & |\arg z|<\frac{\pi}{2}, \\
Y_{\nu}^{1}(z):=\int_{0}^{1}\left(1-t^{2}\right)^{\nu-1 / 2} \sin (z t) d t ; & Y_{\nu}^{2}(z):=\int_{0}^{\infty} e^{-z t}\left(1+t^{2}\right)^{\nu-1 / 2} d t .
\end{array}
$$

Taking into account the analytic continuation formulas [4, Sec. 11, eqs. (10.11.1), (10.11.2)],

$$
\begin{aligned}
& J_{\nu}\left(e^{i m \pi} z\right)=e^{i m \nu \pi} J_{\nu}(z), \quad m=0, \pm 1, \pm 2, \ldots \\
& Y_{\nu}\left(e^{i m \pi} z\right)=e^{-i m \nu \pi} Y_{\nu}(z)+2 i \sin (m \pi \nu) \cot (\pi \nu) J_{\nu}(z),
\end{aligned}
$$

without loss of generality, we may consider the approximation of these functions only for $\Re z \geq 0$.

The power series expansion of (1) (and similarly (2)) may be derived from the integral representation (5) by replacing the cosine in the integrand by its Taylor series expansion 
at the origin and interchanging series and integral. The Taylor expansion converges for $t \in[0,1]$, but the convergence is not uniform in $|z|$. Therefore, the remainders $R_{n}^{(J, 0)}(\nu, z)$ and $R_{n}^{(Y, 0)}(\nu, z)$ are not uniformly bounded in $|z|$.

The asymptotic expansion (3) (and similarly (4)) may be derived from the integral (5) by using Cauchy's theorem to transform the integration path into a vertical line in the complex plane and then applying Watson's lemma [7, Chap. 1, p. 24, Example 4]:

$$
\begin{gathered}
2 \int_{0}^{1}\left(1-t^{2}\right)^{\nu-1 / 2} \cos (z t) d t=\int_{-1}^{1}\left(1-t^{2}\right)^{\nu-1 / 2} \cos (z t) d t= \\
\int_{-1}^{-1+i \infty}\left(1-t^{2}\right)^{\nu-1 / 2} e^{i z t} d t-\int_{1}^{1+i \infty}\left(1-t^{2}\right)^{\nu-1 / 2} e^{i z t} d t
\end{gathered}
$$

After a straightforward change of variables in the last two integrals we get

$$
2 \int_{0}^{1}\left(1-t^{2}\right)^{\nu-1 / 2} \cos (z t) d t=2^{2 \nu}\left[e^{-i(z-\nu \pi / 2-\pi / 4)} I_{-}(z)+e^{i(z-\nu \pi / 2-\pi / 4)} I_{+}(z)\right]
$$

with

$$
I_{ \pm}(z):=\int_{0}^{\infty} t^{\nu-1 / 2}(1 \pm i t)^{\nu-1 / 2} e^{-2 z t} d t
$$

The asymptotic expansion (3) is obtained by applying Watson's lemma to the above integral. Roughly speaking, Watson's lemma consists of a replacement of the factor $(1 \pm i t)^{\nu-1 / 2}$ of the above integrand by its Taylor series at $t=0$ and an interchange of series and integral. Now, the situation with respect to the convergence is worse than in the case of the power series expansion. The Taylor expansion of $(1 \pm i t)^{\nu-1 / 2}$ is not convergent in the whole integration interval $(0, \infty)$, but only in $(0,1)$. This translates into the fact that the expansion that we obtain after interchanging series and integral is not convergent; although it is asymptotic [7, Chap. 1, p. 24, eq. (5.32)]. As the remainder $R_{n}^{(J, \infty)}(\nu, z)$ is unbounded for small $|z|$, the expansion (3) is not uniform in $|z|$. Similarly, the remainder $R_{n}^{(Y, \infty)}(\nu, z)$ is unbounded for small $|z|$ and the expansion (4) is not uniform in $|z|$.

In this paper we propose a different approach that avoids the lack of uniformity in $|z|$ : we consider the Taylor expansion at the origin of the factor $\left(1-t^{2}\right)^{\nu-1 / 2}$ instead of the factor $\cos (z t)$ in (5) or the factor $\sin (z t)$ in (6). This Taylor expansion is convergent for $t$ in the integration interval of the integral (5) and of the first integral (6) and, obviously, it is independent of $z$. After the interchange of series and integral, this fact, the independence of $z$, translates into a remainder that may be bounded independently of $|z|$. The same idea, a Taylor expansion of the factor $\left(1+t^{2}\right)^{\nu-1 / 2}$ in the second integral of $(6)$ does not work as it is not convergent in the whole integration interval. Then, we divide appropriately the integration interval in order to have convergent Taylor expansions of the factor $\left(1+t^{2}\right)^{\nu-1 / 2}$ in both intervals.

As an illustration of the type of approximations that we are going to obtain (see Theorem 2 below), we derive, for example, the following approximation valid for $x>0$ :

$$
\frac{15 \pi}{2 x^{3}} J_{3}(x)=\left[\frac{3 x^{4}-140 x^{2}+360}{8 x^{6}}+\theta_{1}(x)\right] x \sin x+\left[\frac{5\left(x^{2}-18\right)}{2 x^{4}}+\theta_{2}(x)\right] \cos x,
$$


with $\left|\theta_{1}(x)\right|<0.0062$ and $\left|\theta_{2}(x)\right|<0.051$.

The paper is organized as follows. In Theorem 1 in the following section we derive the expansion of $J_{\nu}(z)$ for complex $z$ and $\nu$ with error bounds. We specialize to real $z$ and $\nu$ in Theorem 2, where we obtain more accurate error bounds. In Section 3 we derive the expansion of $Y_{\nu}(z)$ for complex $z$ and $\nu$ with error bounds. Section 4 contains some numerical experiments and a few remarks. Through the paper we use the principal argument $\arg z \in(-\pi, \pi]$ for any complex number $z$ and assume that $\Re \nu>-1 / 2$ and $\Re z \geq 0$. The formulas derived throughout the paper may be extended to $\Re \nu \leq-1 / 2$ using the recurrence relations [4, Sec. 6, eq. 10.6.1],

$$
J_{\nu-1}(z)+J_{\nu+1}(z)=\frac{2 \nu}{z} J_{\nu}(z), \quad Y_{\nu-1}(z)+Y_{\nu+1}(z)=\frac{2 \nu}{z} Y_{\nu}(z) .
$$

\section{A convergent expansion of $J_{\nu}(z)$ uniformly valid in $z$ in any fixed horizontal strip}

A convergent expansion of $J_{\nu}(z)$ in terms of elementary functions and uniformly valid in $z$ in any fixed horizontal strip is given in the following theorem.

Theorem 1. For $n=1,2,3, \ldots$, the Bessel function $J_{\nu}(z)$ may be written in the form:

$$
\frac{\sqrt{\pi} \Gamma(\nu+1 / 2)}{2(z / 2)^{\nu}} J_{\nu}(z)=P_{n-1}(z, \nu) \frac{\sin z}{z}-Q_{n-1}(z, \nu) \cos z+R_{n}(z, \nu),
$$

where $P_{n}(z, \nu)$ and $Q_{n}(z, \nu)$ are the following rational functions:

$$
\begin{array}{ll}
P_{n}(z, \nu):=\sum_{m=0}^{n} \frac{a_{n, m}(\nu)}{\left(-z^{2}\right)^{m}}, & a_{n, m}(\nu):=\sum_{k=m}^{n} \frac{(1 / 2-\nu)_{k}(2 k) !}{k !(2(k-m)) !} \\
Q_{n}(z, \nu):=\sum_{m=1}^{n} \frac{b_{n, m}(\nu)}{\left(-z^{2}\right)^{m}}, & b_{n, m}(\nu):=\sum_{k=m}^{n} \frac{(1 / 2-\nu)_{k}(2 k) !}{k !(2(k-m)+1) !}
\end{array}
$$

and $(\nu)_{k}$ is the Pochhammer symbol: $(\nu)_{k}:=\Gamma(\mu+k) / \Gamma(\mu)$. The functions $P_{n}(z, \nu)$ and $Q_{n}(z, \nu)$ may be computed recursively in the following form:

$$
\begin{array}{ll}
P_{n}(z, \nu)=P_{n-1}(z, \nu)+\frac{(1 / 2-\nu)_{n}(2 n) !}{n !\left(-z^{2}\right)^{n}} c_{n}(z), & P_{0}(z, \nu)=1, \\
Q_{n}(z, \nu)=Q_{n-1}(z, \nu)+\frac{(1 / 2-\nu)_{n}(2 n) !}{n !\left(-z^{2}\right)^{n}} s_{n}(z) & Q_{0}(z, \nu)=0,
\end{array}
$$

with

$$
c_{n}(z):=\sum_{k=0}^{n} \frac{\left(-z^{2}\right)^{k}}{(2 k) !}, \quad s_{n}(z):=\sum_{k=0}^{n-1} \frac{\left(-z^{2}\right)^{k}}{(2 k+1) !}
$$


For $n>\Re \nu-1 / 2$, the remainder $R_{n}(z, \nu)$ is bounded in the form:

$$
\left|R_{n}(z, \nu)\right| \leq \frac{2\left|(1 / 2-\nu)_{n}\right|}{(n-1) !(2 n-1)(2 \Re \nu+1)} e^{|\Im z|}
$$

where $\Im z$ represents the imaginary part of $z$ and ${ }_{3} F_{2}$ is a generalized hypergeometric function [1, Sec. 2, eq. (16.2.1)]. The remainder $R_{n}(z, \nu)$ behaves as $n^{-\Re \nu-1 / 2}$ as $n \rightarrow \infty$ uniformly in $z$ in any fixed horizontal strip. For real $\nu>1 / 2$ and $n \geq \nu-1 / 2$ we also have

$$
\left|R_{n}(z, \nu)\right| \leq \frac{4\left|(1 / 2-\nu)_{n}\right|}{(n-1) !(2 \nu-1)|z|} e^{|\Im z|} .
$$

Proof. Consider the Taylor expansion at the origin of the function $\left(1-t^{2}\right)^{\nu-1 / 2}$ :

$$
\left(1-t^{2}\right)^{\nu-1 / 2}=\sum_{k=0}^{n-1} \frac{(1 / 2-\nu)_{k}}{k !} t^{2 k}+r_{n}(t, \nu), \quad t \in[0,1),
$$

where $r_{n}(t, \nu)$ is the Taylor remainder:

$$
r_{n}(t, \nu):=\sum_{k=n}^{\infty} \frac{(1 / 2-\nu)_{k}}{k !} t^{2 k}, \quad t \in[0,1)
$$

After straightforward manipulations we obtain

$$
\begin{aligned}
r_{n}(t, \nu)= & \frac{(1 / 2-\nu)_{n} t^{2 n}}{n !} \sum_{k=0}^{\infty} \frac{(n+1 / 2-\nu)_{k}}{(n+1)_{k}} t^{2 k} \\
& =\frac{(1 / 2-\nu)_{n} t^{2 n}}{n !}{ }_{2} F_{1}\left(\begin{array}{c}
n+1 / 2-\nu, 1 \\
n+1
\end{array} \mid t^{2}\right), \quad t \in[0,1),
\end{aligned}
$$

where ${ }_{2} F_{1}$ is the Gauss hypergeometric function [3, Sec. 2, eq. (15.2.1)]. Substituting (14) into the integral representation of $\bar{J}_{\nu}(z)$ given in $(5)$ and interchanging sum and integral we obtain

$$
\bar{J}_{\nu}(z)=\sum_{k=0}^{n-1} \frac{(1 / 2-\nu)_{k}}{k !} A_{k}(z)+R_{n}(z, \nu),
$$

with

$$
\begin{aligned}
A_{k}(z):= & \int_{0}^{1} t^{2 k} \cos (z t) d t=(-1)^{k} \frac{d^{2 k}}{d z^{2 k}}\left(\frac{\sin z}{z}\right)= \\
& (-1)^{k} \frac{(2 k) !}{z^{2 k+1}}\left[\sin z \sum_{j=0}^{k} \frac{\left(-z^{2}\right)^{j}}{(2 j) !}-z \cos z \sum_{j=0}^{k-1} \frac{\left(-z^{2}\right)^{j}}{(2 j+1) !}\right]
\end{aligned}
$$

and

$$
R_{n}(z, \nu):=\int_{0}^{1} r_{n}(t, \nu) \cos (z t) d t
$$


When we introduce (17) into (16) and rearrange terms, we obtain (9)-(10).

It is straightforward to see that, for $n=0,1,2, \ldots$, the functions $A_{n}(z)$ satisfy the recurrence relation:

$$
A_{n+1}(z)=\frac{1}{z}\left[\sin z+2(n+1) \frac{\cos z}{z}\right]-\frac{2(n+1)(2 n+1)}{z^{2}} A_{n}(z), \quad A_{0}(z)=\frac{\sin z}{z} .
$$

Recurrences (11) follow from this one after straightforward manipulations.

In order to get the bounds (12) and (13) we write, from (18),

$$
\left|R_{n}(z, \nu)\right| \leq e^{|\Im z|} \int_{0}^{1}\left|r_{n}(t, \nu)\right| d t
$$

Replacing the hypergeometric function in the second line of (15) by its integral representation [3, Sec. 6, eq. (15.6.1)],

$$
{ }_{2} F_{1}\left(\begin{array}{cc|c}
a, & b & \\
c & t^{2}
\end{array}\right)=\frac{\Gamma(c)}{\Gamma(b) \Gamma(c-b)} \int_{0}^{1} u^{b-1}(1-u)^{c-b-1}\left(1-t^{2} u\right)^{-a} d u, \quad \Re c>\Re b>0,
$$

taking the bound $\left|\left(1-t^{2} u\right)^{-a}\right| \leq\left(1-t^{2} u\right)^{-\Re a}$, and using (19) again, we find that, for $n>\Re \nu-1 / 2$,

$$
\left|r_{n}(t, \nu)\right| \leq \frac{\left|(1 / 2-\nu)_{n}\right| t^{2 n}}{n !}{ }_{2} F_{1}\left(\begin{array}{c}
n+1 / 2-\Re \nu, 1 \\
n+1
\end{array} \mid t^{2}\right), \quad t \in[0,1) .
$$

Then,

$$
\begin{aligned}
\left|R_{n}(z, \nu)\right| \leq & e^{|\Im z|} \frac{\left|(1 / 2-\nu)_{n}\right|}{n !} \int_{0}^{1} t_{2}^{2 n} F_{1}\left(\begin{array}{c}
n+1 / 2-\Re \nu, 1 \\
n+1
\end{array} \mid t^{2}\right) d t= \\
& e^{|\Im z|} \frac{\left|(1 / 2-\nu)_{n}\right|}{n !(2 n+1)}{ }_{3} F_{2}\left(\begin{array}{c}
n+1 / 2-\Re \nu, 1, n+1 / 2 \\
n+1, n+3 / 2
\end{array}\right) .
\end{aligned}
$$

From [1, Sec. 4, Eq. (16.4.11)] we have that ${ }_{3} F_{2}\left(\begin{array}{c|c}n+1 / 2-\Re \nu, 1, n+1 / 2 & \\ n+1, n+3 / 2 & 1\end{array}\right)=\frac{\Gamma(n+3 / 2) \Gamma(1 / 2+\Re \nu)}{\Gamma(n+1 / 2) \Gamma(3 / 2+\Re \nu)}{ }_{3} F_{2}\left(\begin{array}{c}1,1 / 2+\Re \nu, 1 / 2 \\ n+1,3 / 2+\Re \nu\end{array}\right)$.

Using the following obvious bound valid for $e>c \geq 0$ and $a, b, d>0$ :

$$
{ }_{3} F_{2}\left(\begin{array}{c|c}
a, b, c & \\
d, e & 1
\end{array}\right) \leq{ }_{2} F_{1}\left(\begin{array}{c|c}
a, b & 1 \\
d &
\end{array}\right)
$$


and formula [3, Sec. 4(ii), eq. (15.4.20)]

$$
{ }_{2} F_{1}\left(\begin{array}{c|c}
a, b & \\
c & 1
\end{array}\right)=\frac{\Gamma(c) \Gamma(c-a-b)}{\Gamma(c-a) \Gamma(c-b)}, \quad \Re(c-a-b)>0,
$$

in the right hand side of (21), and then introducing (21) in (20), we obtain the bound (12).

On the other hand, integrating by parts in (18) we obtain

$$
R_{n}(z, \nu)=r_{n}(1, \nu) \frac{\sin z}{z}-\frac{1}{z} \int_{0}^{1} r_{n}^{\prime}(t, \nu) \sin (z t) d t
$$

It is straightforward to check from (15) that, for real $\nu$ and $n \geq \nu-1 / 2, r_{n}(t, \nu) /(1 / 2-\nu)_{n}$ and $r_{n}^{\prime}(t, \nu) /(1 / 2-\nu)_{n}$ are positive for $t \in[0,1)$. Then,

$$
R_{n}(z, \nu) \leq \frac{\left|(1 / 2-\nu)_{n}\right|}{(1 / 2-\nu)_{n}}\left[r_{n}(1, \nu)+\int_{0}^{1} r_{n}^{\prime}(t, \nu) d t\right] \frac{e^{|\Im z|}}{|z|}=2 \frac{\left|(1 / 2-\nu)_{n}\right|}{(1 / 2-\nu)_{n}} r_{n}(1, \nu) \frac{e^{|\Im z|}}{|z|}
$$

Bound (13) follows from the last line of formula (15), the observation that $r_{n}(t, \nu)$ is continuous at $t=1$ for $\Re \nu>1 / 2$ and (23).

When $z$ is positive and $\nu>-1 / 2$ is real, we can derive more accurate error bounds for the expansion (9) than the ones given in (12) and (13). It is given in Theorem 2. The proof of that theorem requires the following lemma.

Lemma 1. For $x \geq 0$ and $a \geq 1 / 2$,

$$
\left|{ }_{1} F_{2}\left(\begin{array}{c|c}
1 \\
a+2, & a+3 / 2
\end{array} \mid \frac{-x^{2}}{4}\right)\right| \leq 1
$$

and, for $x \geq 1$ and $a \geq 1$,

$$
\mid{ }_{1} F_{2}\left(\begin{array}{c|c}
1 & \frac{-x^{2}}{4} \\
a+2, & a+3 / 2
\end{array} \mid \leq 2(a+1)(2 a+1) \frac{\sigma(x)}{x^{2}},\right.
$$

with $0<\sigma(x) \leq 2$. Numerical experiments suggest that we can replace $\sigma(x)$ by the most accurate bound $1+1 / x$.

Proof. From [6, Sec. 9] we have that

$$
F_{a}(x):={ }_{1} F_{2}\left(\begin{array}{c|c}
1 & -x^{2} \\
a+2, & a+3 / 2
\end{array}\right)=\frac{2(a+1)(2 a+1)}{x^{2 a+3 / 2}} S_{2 a+1 / 2,1 / 2}(x),
$$


where $S_{\mu, \nu}(x)$ is the Lommel's function [6, Sec. 9, eqs. (11.9.3) and (11.9.4)]. Then, from $[2$, eq. (1)], that is valid for $x>0, \nu \geq 0, \mu+\nu>-1$,

$$
\int_{0}^{x} J_{(\mu-\nu+1) / 2}(t) t^{(\nu-\mu+1) / 2}\left(1-\frac{t^{2}}{x^{2}}\right)^{(\mu+\nu-1) / 2} d t=\frac{2^{(\nu-\mu+1) / 2} x^{1-\mu}}{\Gamma((\mu-\nu+1) / 2)} S_{\mu, \nu}(x),
$$

we find the following integral representation valid for $a \geq 1 / 2$ :

$$
\begin{gathered}
F_{a}(x)=\frac{2^{a+3 / 2}(a+1) \Gamma(a+3 / 2)}{x^{2}} \int_{0}^{x} J_{a+1 / 2}(t) t^{1 / 2-a}\left(1-\frac{t^{2}}{x^{2}}\right)^{a} d t \\
=2^{a+3 / 2}(a+1) \Gamma\left(a+\frac{3}{2}\right) \int_{0}^{1} \frac{J_{a+1 / 2}(x t)}{(x t)^{a+1 / 2}} t\left(1-t^{2}\right)^{a} d t .
\end{gathered}
$$

Using formula [4, Sec. 4, eq. (10.14.4)]: $\left|x^{-\nu} J_{\nu}(x)\right| \leq 1 /\left(2^{\nu} \Gamma(\nu+1)\right)$, in the integrand in the second line above, and after some simplifications, we find (24).

Using the formula [4, Sec. 6, eq. (10.6.6)]: $\left(x^{-\nu} J_{\nu}(x)\right)^{\prime}=-x^{-\nu} J_{\nu+1}(x)$, with $\nu=a-1 / 2$, in the integral in the first line of (26), we can integrate by parts to get

$$
F_{a}(x)=\frac{2^{a+3 / 2}(a+1) \Gamma(a+3 / 2)}{x^{2}}\left[\frac{2^{1 / 2-a}}{\Gamma(a+1 / 2)}-2 a \int_{0}^{1} \frac{J_{a-1 / 2}(x t)}{(x t)^{a-1 / 2}} t\left(1-t^{2}\right)^{a-1} d t\right] .
$$

Using again the bound $x^{-\nu} J_{\nu}(x) \geq-1 /\left(2^{\nu} \Gamma(\nu+1)\right)$ in the above integrand we get, after simplifications,

$$
F_{a}(x) \leq \frac{2^{1 / 2-a}}{\Gamma(a+1 / 2)} \sigma(x)
$$

with $\sigma(x)=2$. Numerical experiments suggest that we can replace $\sigma(x)$ by $1+1 / x$. Introducing this bound in the first line of (26) we get (25).

Theorem 2. For $\nu>-1 / 2, x \geq 0$ and $n \geq \max \{1, \nu-1 / 2\}$,

$$
\frac{\sqrt{\pi} \Gamma(\nu+1 / 2)}{2(x / 2)^{\nu}} J_{\nu}(x)=\left[\frac{P_{n-1}(x, \nu)}{x^{2}}+R_{n}^{P}(x, \nu)\right] x \sin x-\left[Q_{n-1}(x, \nu)+R_{n}^{Q}(x, \nu)\right] \cos x,
$$

where $P_{n}(z, \nu)$ and $Q_{n}(z, \nu)$ are the rational functions given in Theorem 1 . Write $m:=$ $\lfloor\nu-1 / 2\rfloor$ and $\alpha:=\nu-1 / 2-m$, where the symbol $\lfloor\nu\rfloor$ stands for the biggest integer smaller than or equal to $\nu$. Then, the remainders $R_{n}^{P}(x, \nu)$ and $R_{n}^{Q}(x, \nu)$ are uniformly bounded in the form:

$$
\left|R_{n}^{P}(x, \nu)\right| \leq \frac{(\alpha)_{m+1}(1-\alpha)_{n-m-1}}{n !(2 n-1)(2 \nu+3)}
$$

and

$$
\left|R_{n}^{Q}(x, \nu)\right| \leq \frac{2(\alpha)_{m+1}(1-\alpha)_{n-m-1}}{(n-1) !(2 n-1)(2 \nu+1)}
$$


Also, for $\nu>3 / 2$ and $x \geq 1$, the remainders are bounded in the form:

$$
\left|R_{n}^{P}(x, \nu)\right| \leq \frac{2(\alpha)_{m+1} \Gamma(n-\nu+1 / 2)}{(2 \nu-1) \Gamma(1-\alpha)(n-1) !} \frac{\sigma(x)}{x^{2}}
$$

and

$$
\left|R_{n}^{Q}(x, \nu)\right| \leq \frac{4(\alpha)_{m+1} \Gamma(n-\nu+1 / 2)}{(2 \nu-3) \Gamma(1-\alpha)(n-2) !} \frac{\sigma(x)}{x^{2}},
$$

with $1+\frac{1}{x} \leq \sigma(x) \leq 2$.

Proof. We use that

$$
\sum_{k=0}^{n} \frac{\left(-z^{2}\right)^{k}}{(2 k) !}=\cos z+\frac{(-1)^{n} z^{2 n+2}}{(2 n+2) !}{ }_{1} F_{2}\left(\begin{array}{c|c}
1 \\
n+2, & n+3 / 2
\end{array} \mid-\frac{z^{2}}{4}\right)
$$

and

$$
\sum_{k=0}^{n-1} \frac{\left(-z^{2}\right)^{k}}{(2 k+1) !}=\frac{\sin z}{z}-\frac{(-1)^{n} z^{2 n}}{(2 n+1) !} F_{2}\left(\begin{array}{c|c}
1 \\
n+1, n+3 / 2
\end{array} \mid-\frac{z^{2}}{4}\right)
$$

in the second line of (17). Rearranging terms we find, after straightforward computations, that $\bar{J}_{\nu}(x)$ may be written in the form $(27)$ with

$$
\begin{gathered}
R_{n}^{P}(x, \nu):=\sum_{k=n}^{\infty} \frac{(1 / 2-\nu)_{k}}{k !(2 k+1)(2 k+2)}{ }_{1} F_{2}\left(\begin{array}{c|c}
1 \\
k+2, & k+3 / 2
\end{array} \mid-\frac{x^{2}}{4}\right), \\
R_{n}^{Q}(x, \nu):=-\sum_{k=n}^{\infty} \frac{(1 / 2-\nu)_{k}}{k !(2 k+1)}{ }_{1} F_{2}\left(\begin{array}{c|c}
1 \\
k+1, k+3 / 2
\end{array} \mid-\frac{x^{2}}{4}\right) .
\end{gathered}
$$

We will find bounds for these two series by using Lemma 1 and also the fact that, for $k \geq m+1(n \geq \nu-1 / 2)$ and $\alpha:=\nu-1 / 2-m$,

$$
(1 / 2-\nu)_{k}=(-1)^{m}(\alpha)_{m+1}(1-\alpha)_{k-m-1} .
$$

From this identity and bound (24) with $a=k$ or $a=k-1 / 2$ we find that, for $n \geq 1$,

$$
\begin{aligned}
\left|R_{n}^{P}(x, \nu)\right| \leq & (\alpha)_{m+1} \sum_{k=n}^{\infty} \frac{(1-\alpha)_{k-m-1}}{k !(2 k+1)(2 k+2)}= \\
& \frac{(\alpha)_{m+1}(1-\alpha)_{n-m-1}}{n !(2 n+1)(2 n+2)}{ }_{3} F_{2}\left(\begin{array}{cc|c}
n+1 / 2, n-m-\alpha, & 1 \\
n+3 / 2, n+2 & 1
\end{array}\right)
\end{aligned}
$$


and

$$
\begin{aligned}
\left|R_{n}^{Q}(x, \nu)\right| \leq & (\alpha)_{m+1} \sum_{k=n}^{\infty} \frac{(1-\alpha)_{k-m-1}}{k !(2 k+1)}= \\
& \frac{(\alpha)_{m+1}(1-\alpha)_{n-m-1}}{n !(2 n+1)}{ }_{3} F_{2}\left(\begin{array}{c}
n+1 / 2, n-m-\alpha, 1 \\
n+3 / 2, n+1
\end{array} \mid 1\right) .
\end{aligned}
$$

Bounds (28) and (29) follow from these inequalities, formula [1, Sec. 4, Eq. (16.4.11)], inequality (22) and formula (23).

From (32) and bound (25) with $a=k$ or $a=k-1 / 2$ we find that

$$
\left|R_{n}^{P}(x, \nu)\right| \leq \sigma \frac{(\alpha)_{m+1}}{x^{2}} \sum_{k=n}^{\infty} \frac{(1-\alpha)_{k-m-1}}{k !}=\frac{2(\alpha)_{m+1} \Gamma(n-\nu+1 / 2)}{(2 \nu-1) \Gamma(1-\alpha)(n-1) !} \frac{\sigma(x)}{x^{2}}
$$

and

$$
\left|R_{n}^{Q}(x, \nu)\right| \leq 2 \sigma \frac{(\alpha)_{m+1}}{x^{2}} \sum_{k=n}^{\infty} \frac{(1-\alpha)_{k-m-1}}{(k-1) !}=\frac{4(\alpha)_{m+1} \Gamma(n-\nu+1 / 2)}{(2 \nu-3) \Gamma(1-\alpha)(n-2) !} \frac{\sigma(x)}{x^{2}}
$$

Formula (7) is a rewriting of formulas (27)-(29) for $\nu=n=3$. From (28) we find that $R_{3}^{P}(x, 3) \leq 0.00615$ and from $(29)$ we find that $R_{3}^{Q}(x, 3) \leq 0.0508$.

\section{A convergent expansion of $Y_{\nu}(z)$ uniformly valid in $z$ in any fixed horizontal strip}

The expansion of $Y_{\nu}(z)$ is derived in Theorem 3 below. It is achieved in three steps detailed in the respective propositions 1, 2 and 3. In Proposition 1 we derive an expansion for $Y_{\nu}^{1}(z)$; the derivation is similar to that of $\bar{J}_{\nu}(z)$ in Theorem 1 , just replacing $\cos (z t)$ by $\sin (z t)$ in the proof of that theorem. The expansion of $Y_{\nu}^{2}(z)$ is a little bit more cumbersome as the integration interval is unbounded and then, just the use of the Taylor expansion of $\left(1+t^{2}\right)^{\nu-1 / 2}$ does not work. To overcome this problem we write $Y_{\nu}^{2}(z)=F_{\nu}(z)+G_{\nu}(z)$ with

$$
F_{\nu}(z):=\int_{0}^{1} e^{-z t}\left(1+t^{2}\right)^{\nu-1 / 2} d t, \quad G_{\nu}(z):=\int_{1}^{\infty} e^{-z t}\left(1+t^{2}\right)^{\nu-1 / 2} d t,
$$

and approximate both integrals independently in propositions 2 and 3 respectively.

Proposition 1. For $n=1,2,3, \ldots$, the function $Y_{\nu}^{1}(z)$ may be written in the form:

$$
Y_{\nu}^{1}(z)=S_{n-1}(z, \nu) \frac{1}{z}-P_{n-1}(z, \nu) \frac{\cos z}{z}-Q_{n-1}(z, \nu) \sin z+R_{n}^{1}(z, \nu),
$$


where $P_{n}(z, \nu)$ and $Q_{n}(z, \nu)$ are given in Theorem 1 and $S_{n}(z, \nu)$ is the following rational function:

$$
S_{n}(z, \nu):=\sum_{k=0}^{n} \frac{(1 / 2-\nu)_{k}(2 k) !}{k !\left(-z^{2}\right)^{k}} .
$$

The remainder $R_{n}^{1}(z, \nu)$ has the same bounds (12) and (13) as the remainder $R_{n}(z, \nu)$ in Theorem 1 and under the same conditions.

Proof. It is identical to the proof of Theorem 1, except for the fact that now, equation (17) reads

$$
\begin{aligned}
A_{n}(z, \nu):= & \int_{0}^{1} t^{2 n} \sin (z t) d t=(-1)^{n} \frac{d^{2 n}}{d z^{2 n}}\left(\frac{1-\cos z}{z}\right)= \\
& (-1)^{n} \frac{(2 n) !}{z^{2 n+1}}\left[\frac{1}{z}-\cos z \sum_{k=0}^{n} \frac{\left(-z^{2}\right)^{k}}{(2 k) !}-z \sin z \sum_{k=0}^{n-1} \frac{\left(-z^{2}\right)^{k}}{(2 k+1) !}\right]
\end{aligned}
$$

and

$$
R_{n}^{1}(z, \nu):=\int_{0}^{1} r_{n}(t, \nu) \sin (z t) d t
$$

Proposition 2. For $n=1,2,3, \ldots$, the function $F_{\nu}(z)$ may be written in the form:

$$
F_{\nu}(z)=S_{n-1}(z, \nu) \frac{1}{z}-T_{n-1}(z, \nu) \frac{e^{-z}}{z}+R_{n}^{F}(z, \nu)
$$

where $S_{n}(z, \nu)$ is given in (35) and $T_{n}(z, \nu)$ is the following rational function:

$$
T_{n}(z, \nu):=\sum_{k=0}^{n} \frac{(1 / 2-\nu)_{k}(2 k) !}{k !\left(-z^{2}\right)^{k}} e_{2 k}(z), \quad e_{k}(z):=\sum_{j=0}^{k} \frac{z^{j}}{j !}=\frac{e^{z}}{k !} \Gamma(k+1, z) .
$$

For $n>\Re \nu-1 / 2$, the remainder term may be bounded in the following forms:

$$
\left|R_{n}^{F}(z, \nu)\right| \leq \frac{\left|(1 / 2-\nu)_{n}\right|}{(2 n+1) n !} \quad \text { and } \quad\left|R_{n}^{F}(z, \nu)\right| \leq \frac{\left|(1 / 2-\nu)_{n}\right|}{n ! \Re z}\left(1-e^{-\Re z}\right) .
$$

Proof. It is similar to the proof of Theorem 1. Consider the Taylor expansion at the origin of the function $\left(1+t^{2}\right)^{\nu-1 / 2}$ :

$$
\left(1+t^{2}\right)^{\nu-1 / 2}=\sum_{k=0}^{n-1} \frac{(1 / 2-\nu)_{k}}{k !}\left(-t^{2}\right)^{k}+r_{n}^{F}(t, \nu), \quad t \in[0,1),
$$

where $r_{n}^{F}(t, \nu)$ is the Taylor remainder:

$$
r_{n}^{F}(t, \nu):=\sum_{k=n}^{\infty} \frac{(1 / 2-\nu)_{k}}{k !}\left(-t^{2}\right)^{k}, \quad t \in[0,1) .
$$


After straightforward manipulations we obtain

$$
r_{n}^{F}(t, \nu)=\frac{(1 / 2-\nu)_{n}\left(-t^{2}\right)^{n}}{n !}{ }_{2} F_{1}\left(\begin{array}{c|c}
n+1 / 2-\nu, & 1 \\
n+1 & -t^{2}
\end{array}\right), \quad t \in[0,1) .
$$

Substituting (39) into (33) and interchanging sum and integral we obtain

$$
F_{\nu}(z)=\sum_{k=0}^{n-1} \frac{(1 / 2-\nu)_{k}}{k !}(-1)^{k} C_{k}(z, \nu)+R_{n}^{F}(z, \nu)
$$

with

$$
C_{n}(z, \nu):=\int_{0}^{1} t^{2 n} e^{-z t} d t=\frac{d^{2 n}}{d z^{2 n}}\left(\frac{1-e^{-z}}{z}\right)=\frac{(2 n) !}{z^{2 n+1}}\left[1-e^{-z} \cdot e_{2 n}(z)\right]
$$

and

$$
R_{n}^{F}(z, \nu):=\int_{0}^{1} r_{n}^{F}(t, \nu) e^{-z t} d t
$$

Introducing (42) in (41) and rearranging terms we obtain (36)-(37).

For $n \geq \Re \nu-1 / 2$ and $t \in[0,1]$, we derive easily from the integral representation (19) that the hypergeometric function in (40) is bounded by 1 . Therefore,

$$
\left|R_{n}^{F}(z, \nu)\right| \leq \frac{\left|(1 / 2-\nu)_{n}\right|}{n !} \int_{0}^{1} t^{2 n} e^{-t \Re z} d t
$$

The first bound in (38) follows from this equation straightforwardly. Integrating by parts in the above integral we obtain the second bound in (38).

In general, for arbitrary values of $\nu$, we have no means to approximate $G_{\nu}(z)$ uniformly in $z$ in any fixed horizontal strip in terms of elementary functions, as we did for $J_{\nu}(z), Y_{\nu}^{1}(z)$ and $F_{\nu}(z)$. But it is possible when $\nu$ is a positive integer. In the following proposition we give a uniform approximation of $G_{\nu}(z)$ for general values of $\nu$ in terms of incomplete gamma functions. In Corollary 1 we give a uniform approximation of $G_{m}(z)$ for $m \in N$ (natural numbers) in terms of elementary functions.

Proposition 3. For $n=1,2,3, \ldots$, the function $G_{\nu}(z)$ may be written in the form:

$$
G_{\nu}(z)=\frac{1}{z^{2 \nu}} \sum_{k=0}^{n-1} \frac{(1 / 2-\nu)_{k}}{k !}\left(-z^{2}\right)^{k} \Gamma(2 \nu-2 k, z)+R_{n}^{G}(z, \nu)
$$

where, for $n>\Re \nu$, the remainder term may be bounded in the form

$$
\left|R_{n}^{G}(z, \nu)\right| \leq \frac{\left|(1 / 2-\nu)_{n}\right|}{2(n-\Re \nu) n !} e^{-\Re z} .
$$

Also, for $n>\Re \nu-1 / 2$ it is bounded in the form

$$
\left|R_{n}^{G}(z, \nu)\right| \leq \frac{2\left|(1 / 2-\nu)_{n}\right|}{n ! \Re z} e^{-\Re z} .
$$


Proof. The integral defining $G_{\nu}(z)$ in (33) may be written in the form

$$
G_{\nu}(z):=\int_{1}^{\infty} e^{-z t} t^{2 \nu-1}\left(1+t^{-2}\right)^{\nu-1 / 2} d t
$$

Then, we consider the Taylor expansion at the infinity of the function $\left(1+t^{-2}\right)^{\nu-1 / 2}$ :

$$
\left(1+t^{-2}\right)^{\nu-1 / 2}=\sum_{k=0}^{n-1} \frac{(1 / 2-\nu)_{k}}{k !}\left(-t^{2}\right)^{-k}+r_{n}^{G}(t, \nu), \quad t \in[1, \infty),
$$

where $r_{n}^{G}(t, \nu)$ is the Taylor remainder:

$$
r_{n}^{G}(t, \nu):=\sum_{k=n}^{\infty} \frac{(1 / 2-\nu)_{k}}{k !}\left(-t^{2}\right)^{-k}, \quad t \in[1, \infty) .
$$

After straightforward manipulations we obtain

$$
r_{n}^{G}(t, \nu)=\frac{(1 / 2-\nu)_{n}}{n !\left(-t^{2}\right)^{n}}{ }_{2} F_{1}\left(\begin{array}{c}
n+1 / 2-\nu, 1 \\
n+1
\end{array} \mid-\frac{1}{t^{2}}\right), \quad t \in[1, \infty) .
$$

Substituting (47) into (46) and interchanging sum and integral we obtain (43) with

$$
R_{n}^{G}(z, \nu):=\int_{1}^{\infty} t^{2 \nu-1} r_{n}^{G}(t, \nu) e^{-z t} d t
$$

For $n \geq \Re \nu-1 / 2$ and $t \in[1, \infty)$, we derive easily from the integral representation (19), that the hypergeometric function in (49) is bounded by 1 . Therefore,

$$
\left|R_{n}^{G}(z, \nu)\right| \leq \frac{\left|(1 / 2-\nu)_{n}\right|}{n !} \int_{1}^{\infty} t^{2 \Re \nu-2 n-1} e^{-t \Re z} d t .
$$

Bound (44) follows from this equation straightforwardly. Integrating by parts in the above integral we obtain the bound (45).

Observation 1. The terms of the expansion (43) may be computed recursively [5, Sec. 8, eq. (8.8.2)] starting from $\Gamma(2 \nu, z): \Gamma(a+1, z)=a \Gamma(a, z)+z^{a} e^{-z}$. For $n=0,1,2, \ldots$ and $\nu \notin Z$ (integer numbers), the incomplete gamma functions satisfy the recurrence relation:

$$
\Gamma(2 \nu-2 n-2, z)=\frac{\Gamma(2 \nu-2 n, z)+(2 n+1-2 \nu-z) z^{2 \nu-2 n-2} e^{-z}}{2(\nu-n-1)(2 \nu-2 n-1)} .
$$

From propositions 1, 2 and 3 we find the following expansion of the Bessel function $Y_{\nu}(z)$ uniformly valid in $z$ in any fixed horizontal strip: 
Theorem 3. For $n=1,2,3, \ldots$, the Bessel function $Y_{\nu}(z)$ may be written in the form:

$$
\begin{gathered}
\frac{\sqrt{\pi} \Gamma(\nu+1 / 2)}{2(z / 2)^{\nu}} Y_{\nu}(z)=\sum_{k=0}^{n-1}\left\{\frac{(1 / 2-\nu)_{k}}{k !}\left[\frac{\Gamma(2 k+1, z)}{z\left(-z^{2}\right)^{k}}-\frac{\left(-z^{2}\right)^{k} \Gamma(2 \nu-2 k, z)}{z^{2 \nu}}\right]-\right. \\
\left.\frac{a_{n-1, k}(\nu) \cos z+b_{n-1, k}(\nu) z \sin z}{z\left(-z^{2}\right)^{k}}\right\}+R_{n}^{Y}(z, \nu),
\end{gathered}
$$

with

$$
a_{n-1, k}(\nu):=\sum_{j=k}^{n-1} \frac{(1 / 2-\nu)_{j}(2 j) !}{j !(2(j-k)) !}, \quad b_{n-1, k}(\nu):=\sum_{j=k}^{n-1} \frac{(1 / 2-\nu)_{j}(2 j) !}{j !(2(j-k)+1) !},
$$

for $k=0,1,2, \ldots, n$, except $b_{n-1,0}(\nu)=0$. For $n>\Re \nu$, the remainder $R_{n}^{Y}(z, \nu)$ is bounded in the form:

$$
\left|R_{n}^{Y}(z, \nu)\right| \leq \frac{\left|(1 / 2-\nu)_{n}\right|}{n !}\left[\frac{2 n e^{|\Im z|}}{(2 n-1)(2 \Re \nu+1)}+\frac{1}{2 n+1}+\frac{e^{-\Re z}}{2(n-\Re \nu)}\right],
$$

and behaves as $n^{-\Re \nu-1 / 2}$ as $n \rightarrow \infty$ uniformly in $z$ in any fixed horizontal strip. For real $\nu>1 / 2$ and $n>\nu-1 / 2$ it is bounded in the form:

$$
\left|R_{n}^{Y}(z, \nu)\right| \leq \frac{\left|(1 / 2-\nu)_{n}\right|}{n !}\left[\frac{4 n e^{|\Im z|}}{(2 \nu-1)|z|}+\frac{1+e^{-\Re z}}{\Re z}\right] .
$$

Observation 2. When $\nu=m \in N$ we have that recurrence (51) is valid for $n \geq m$. On the other hand, from the definition [5, Sec. 2, eq. (8.2.2)],

$$
\Gamma(a, z):=\int_{z}^{\infty} t^{a-1} e^{-t} d t
$$

we find that, for $n=0,1,2, \ldots, m-1$,

$$
\Gamma(2 m-2 n, z)=-z^{2 m-2 n} \frac{d^{2 m-2 n-1}}{d z^{2 m-2 n-1}}\left(\frac{e^{-z}}{z}\right)=(2 m-2 n-1) ! e^{-z} e_{2 m-2 n-1}(z),
$$

with $e_{k}(z)$ given in (37). Therefore, when $\nu=m \in N$, the $m$-th order approximation of $Y_{m}(z)$ given in Theorem 3 is given in terms of elementary functions.

\section{Final remarks and numerical experiments}

Observe that the function $Y_{\nu}^{1}(z)$ defined in (6) is related to the Struve function $\mathbf{H}_{\nu}(z)[6$, Sec. 5, eq. (11.5.1)]:

$$
\mathbf{H}_{\nu}(z)=\frac{2^{1-\nu} z^{\nu}}{\sqrt{\pi} \Gamma(\nu+1 / 2)} Y_{\nu}^{1}(z)
$$


Therefore, the expansion (34) multiplied by the above factor is a convergent expansion of $\mathbf{H}_{\nu}(z)$ uniformly in $z$ in any fixed horizontal strip.

From the recurrence relations (8) and the special values [4, Sec. 16, eqs. (10.16.1)],

$$
J_{1 / 2}(z)=Y_{-1 / 2}(z)=\sqrt{\frac{2}{\pi z}} \sin z, \quad J_{-1 / 2}(z)=-Y_{1 / 2}(z)=\sqrt{\frac{2}{\pi z}} \cos z,
$$

we know that, for half-integer values of $\nu, J_{\nu}(z)$ and $Y_{\nu}(z)$ are elementary functions of $z$. This is confirmed by all the expansions given in the above theorems: when $\nu=k+1 / 2$, $k=0,1,2, \ldots$, the presence of the factor $(1 / 2-\nu)_{n}$ in the bounds for the remainders $R_{n}(z, \nu)$ shows that the remainders vanish for $n \geq k+1$, and then the approximations are exact (and given in terms of elementary functions). More precisely, for half-integer values of $\nu$ :

$$
J_{\nu}(z)=\frac{2(z / 2)^{\nu}}{\sqrt{\pi} \Gamma(\nu+1 / 2)}\left[1+\frac{d^{2}}{d z^{2}}\right]^{\nu-1 / 2} \frac{\sin z}{z}
$$

and

$$
Y_{\nu}(z)=-\frac{2(z / 2)^{\nu}}{\sqrt{\pi} \Gamma(\nu+1 / 2)}\left[1+\frac{d^{2}}{d z^{2}}\right]^{\nu-1 / 2} \frac{\cos z}{z} .
$$

From the Poisson's integral representations (5) and (6) we see that these expressions are formally valid for any real $\nu$. This is so because when we replace $t^{2} \cos (z t)$ by $-d^{2} \cos (z t) / d z^{2}$ in (5) we find

$$
\int_{0}^{1}\left(1-t^{2}\right)^{\nu-1 / 2} \cos (z t) d t=\left[1+\frac{d^{2}}{d z^{2}}\right]^{\nu-1 / 2} \int_{0}^{1} \cos (z t) d t=\left[1+\frac{d^{2}}{d z^{2}}\right]^{\nu-1 / 2} \frac{\sin z}{z}
$$

And similarly when we replace $t^{2} \sin (z t)$ by $-d^{2} \sin (z t) / d z^{2}$ in the first integral of (6) and $t^{2} e^{-z t}$ by $d^{2} e^{-z t} / d z^{2}$ in the second integral of (6), we find:

$$
\left[1+\frac{d^{2}}{d z^{2}}\right]^{\nu-1 / 2} \frac{1-\cos z}{z} \text { and } \quad-\left[1+\frac{d^{2}}{d z^{2}}\right]^{\nu-1 / 2} \frac{1}{z} \quad \text { respectively. }
$$

Therefore, for half-integer values of $\nu, z^{-\nu} J_{\nu}(z)$ is an elementary function of $z$. For other values of $\nu$ it is not, but at least, it is an infinite expansion of elementary functions of $z$ uniformly valid in $z$ in any fixed horizontal strip.

Figures 1 and 2 illustrate the accuracy of the expansions given in theorems 1 and 2 for $J_{\nu}(z)$, with $\nu=2$, several values of $z$ and different orders $n$ of the approximation; the situation is similar for other values of $\nu$. The accuracy of the expansion given in theorem 3 for $Y_{\nu}(z)$ is similar. The Figures have been obtained by using the program Mathematica 7.

The convergent expansions of the Bessel functions given in (9) and (52) are alternative formulas to the convergent power expansions given in (1) and (2), or to the asymptotic 

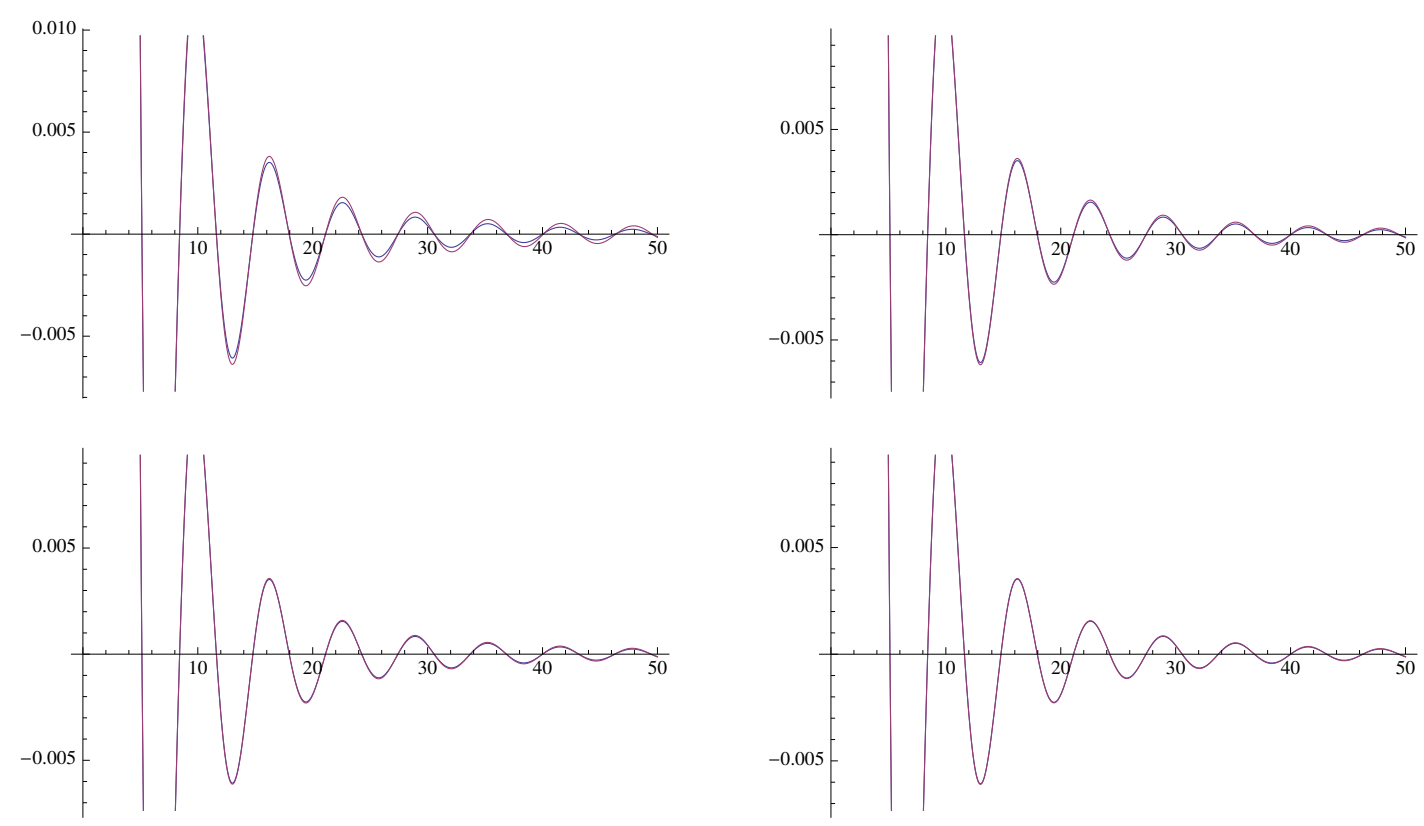

Figure 1: Plot of the function $\bar{J}_{2}(x)$ and the right hand side of (16) for $n=10, n=15$ (top) and $n=20, n=25$ (bottom) in the real interval $[0,50]$.
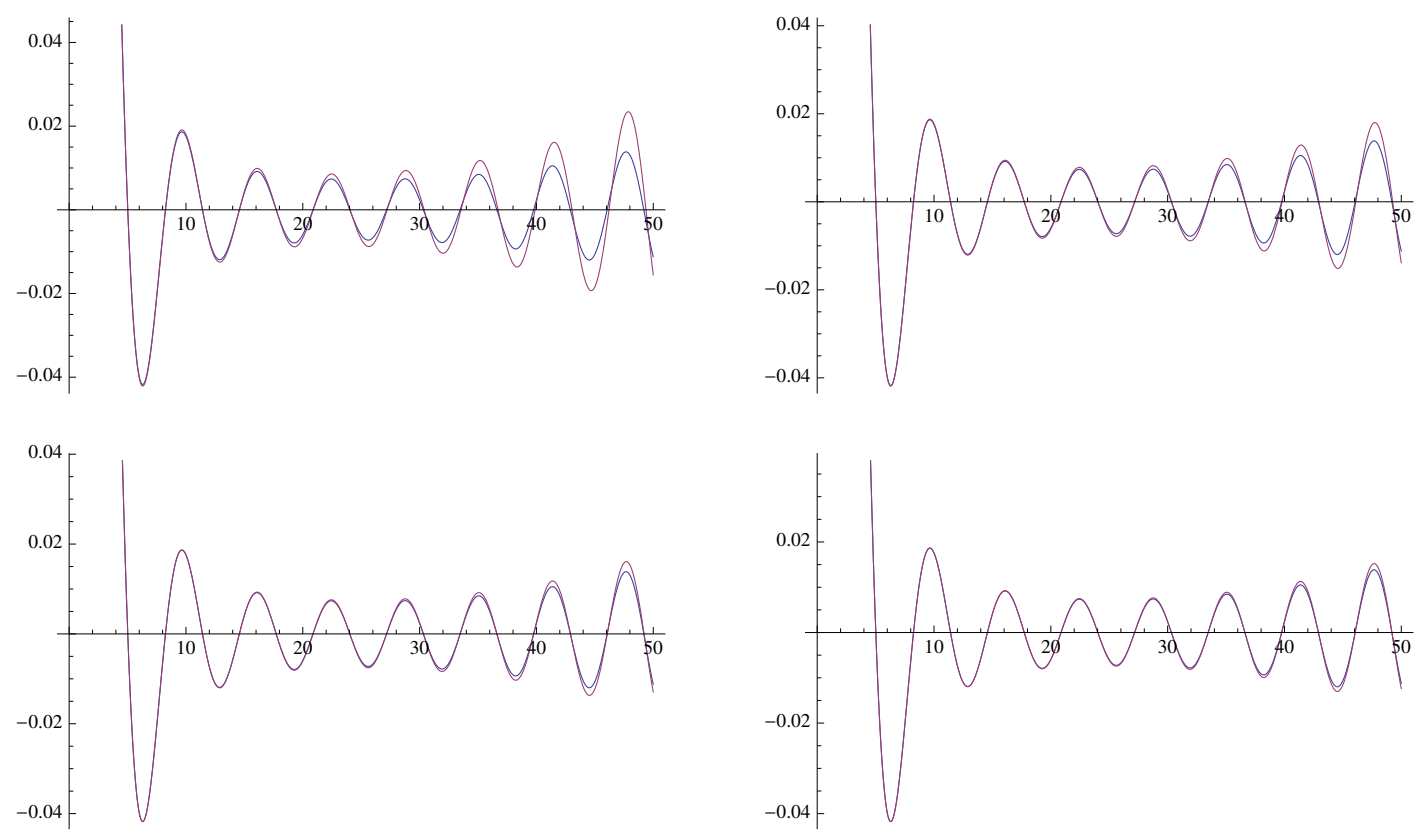

Figure 2: Plot of the real part of the function $\bar{J}_{2}((1+0.1 i) x)$ and the real part of the right hand side of (16) for $n=10, n=15$ (top) and $n=20, n=25$ (bottom) for $0<x<50$. 

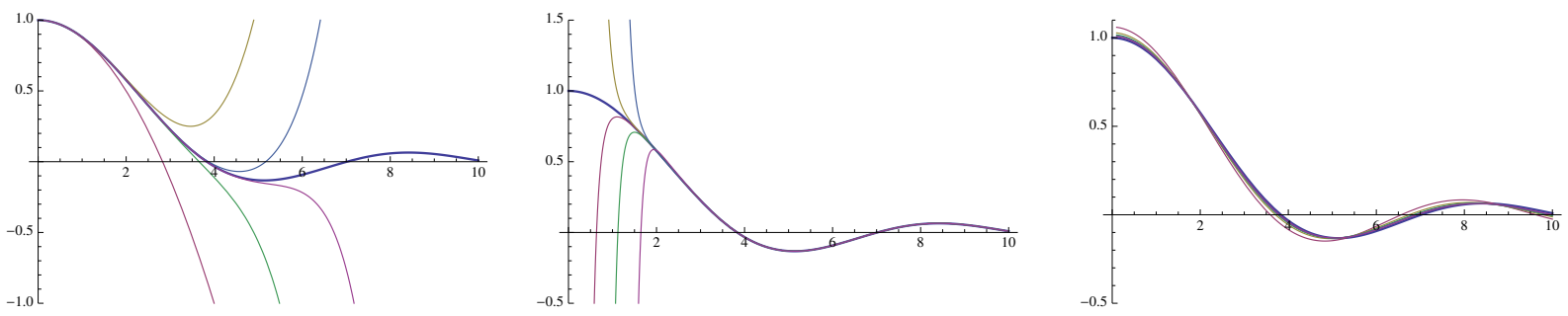

Figure 3: Approximations of $(2 / z)^{\nu} J_{\nu}(z)$ (thicker graphics) given by the expansions (1) (left), (3) (middle) and (9) (right) for $\nu=1, z \in[0,10]$ and five degrees of approximation $n=1,2,3,4,5$ (thinner graphics). The approximations are similar for complex $z$ and other values of $\nu$.

inverse power expansions given in (3) and (4). All of these expansions are given in terms of elementary functions, but the character of the approximation is different. At a given order $n$ of the approximation, the remainder terms in the power expansions (1) and (2) are proportional to $|z|^{n}$; the remainder terms in the asymptotic expansions (3) and (4) are proportional to $|z|^{-n}$. On the other hand, the remainder terms of the expansions given in theorems 1 or 2 and in Theorem 3 are independent of $z$ in any fixed horizontal strip. Therefore, when we want to compute the Bessel functions within a prescribed accuracy with (1) or (2), the number of terms $n$ needed increases as $|z|$ increases. When we want to compute the Bessel functions within a prescribed accuracy with (3) or (4), the number of terms $n$ needed increases as $|z|$ decreases; or even worse, it is not possible to get that accuracy for any value of $n$. On the other hand, the number of terms $n$ required to compute the Bessel functions within a prescribed accuracy using theorems 1,2 or 3 is fixed independently of $z$ in any fixed horizontal strip. Figures 3 and 4 illustrate these facts for the Bessel function $J_{\nu}(z)$; the situation is similar for the approximations (2), (4) and (52) of the Bessel function $Y_{\nu}(z)$. Then, in general, expansions (1) and (2) are more accurate for small $|z|$ and expansions (3) and (4) are more accurate for large $|z|^{1}$. On the other hand, expansions (9) and (52) are more uniform approximations in $|z|$ in any fixed horizontal strip.

\section{Acknowledgments}

This research was supported by the Spanish Ministry of "Economía y Competitividad", project MTM2014-52859-P. The Universidad Pública de Navarra is acknowledged by its financial support. Professor Dmitrii Karp is acknowledged by his helpful comments.

\section{References}

[1] R. A. Askey and A. B. Olde Daalhuis, Generalized Hypergeometric Functions and Meijer G-Function, in: NIST Handbook of Mathematical Functions, Cambridge University Press, Cambridge, 2010, pp. 403-418

\footnotetext{
${ }^{1}$ Although from the numerical experiments we observe that, for certain values of $\nu$ and $n$, expansions (9) and (52) are more accurate for certain (moderate) values of $|z|$.
} 

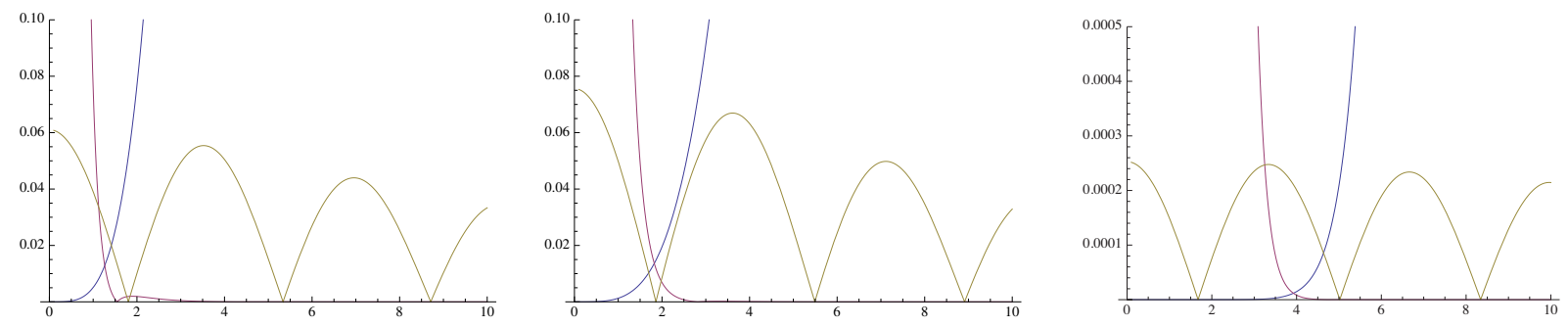

Figure 4: Absolute value of the absolute error in the approximation of $(2 / z)^{\nu} J_{\nu}(z)$ in the interval $z \in[0,10]$ given by the expansions (1) (blue), (3) (red) and (9) (gold) with $n=\nu=1$ (left), $n=1$ and $\nu=2$ (middle) and $n=\nu=3$ (right). The behavior is similar for complex $z$ and other values of $n$ and $\nu$.

(Chapter 16).

[2] R. G. Cooke, On the sign of Lommel's function, J. London Math. Soc. s1-7 (4) (1932) 281-283.

[3] A. B. Olde Daalhuis, Hypergeometric Function, in: NIST Handbook of Mathematical Functions, Cambridge University Press, Cambridge, 2010, pp. 383-401 (Chapter 15).

[4] F. W. J. Olver and L. C. Maximon, Bessel Functions, in: NIST Handbook of Mathematical Functions, Cambridge University Press, Cambridge, 2010, pp. 215-286 (Chapter 10).

[5] R. B. Paris, Incomplete Gamma and Related Functions, in: NIST Handbook of Mathematical Functions, Cambridge University Press, Cambridge, 2010, pp. 173-192 (Chapter 8).

[6] R. B. Paris, Struve and Related Functions, in: NIST Handbook of Mathematical Functions, Cambridge University Press, Cambridge, 2010, pp. 287-302 (Chapter 11).

[7] R. Wong, Asymptotic approximations of integrals, Academic Press, New York, 1989. 